\title{
The L-type lectin LMAN2 is over-expressed in brain metastatic breast cancer.
}

Shahan Mamoor, $\mathrm{MS}^{1}$

1shahanmamoor@gmail.com

East Islip, NY USA

One study reported the incidence of central nervous system metastases in breast cancer patients treated with trastuzumab as $34 \%{ }^{1}$. We mined published microarray data ${ }^{2,3}$ to discover genes associated with brain metastasis in breast cancer. We identified significant differential expression of the L-type lectin LMAN24-7, also known as VIP36, in patients with breast cancer. LMAN2 may be relevant to the biology underlying colonization of the brain with metastatic breast cancer clones.

Keyword: breast cancer, metastasis, brain metastasis, central nervous system metastasis, LMAN2, VIP36, systems biology of breast cancer, targeted therapeutics in breast cancer. 
One study reported a $34 \%$ incidence of central nervous system metastases in patients with breast cancer treated with the monoclonal antibody trastuzumab (Herceptin) ${ }^{1}$. Treatment options for patients with brain metastatic breast cancer are limited ${ }^{8}$. We mined published microarray datasets ${ }^{2-3}$ to understand in an unbiased and systems-level fashion genes whose expression most significantly distinguished brain metastases from primary tumors of the breast, the cells from which these metastatic clones are presumably derived. We found significant differential expression of the L-type lectin LMAN24-7 in the brain metastases of patients with brain metastatic breast cancer.

\section{Methods}

We utilized microarray datasets GSE $100534^{2}$ and GSE438373 for this differential gene expression analysis of brain metastatic breast cancer in conjunction with GEO2R. GSE100534 was generated using Affymetrix Human Gene 1.0 ST Array technology with $n=16$ primary tumors from patients with breast cancer and $n=3$ brain metastases from patients with brain metastatic breast cancer. GSE43837 was generated using Affymetrix Human X3P Array technology with $n=19$ primary breast tumors and $n=19$ brain metastases from patients with breast cancer, analysis performed using platform GPL1352. The Benjamini and Hochberg method of $p$-value adjustment was used for ranking of differential expression but raw $p$-values were used to assess statistical significance of global differential expression. Logtransformation of data was auto-detected, and the NCBI generated category of platform annotation was used. A statistical test was performed to evaluate whether LMAN2 gene expression was significantly between primary breast tumors and brain metastases in humans with breast cancer using a two-tailed, unpaired t-test with Welch's correction. We used PRISM for all statistical analyses of differential gene expression in human breast cancer (Version 8.4.0)(455).

\section{Results}

We mined published microarray data ${ }^{2,3}$ to discover genes associated with brain metastasis in breast cancer.

\section{LMAN2 is differentially expressed in brain metastatic breast cancer.}

We identified LMAN2 as among the genes whose expression changes most significantly when comparing brain metastases to primary tumors of the breast in patients with brain metastatic breast cancer $^{2}$ (Table 1). When sorting each of the genes expressed in brain metastases based on significance of change as compared to primary tumors of the breast, LMAN2 ranked 87 out of 33297 total transcripts (Table 1). Differential expression of LMAN2 in brain metastases in brain metastatic breast cancer was statistically significant (Table $1 ; \mathrm{p}=4.99 \mathrm{E}-07$ ).

We queried a second microarray dataset ${ }^{3}$ to validate transcriptome-wide differential expression of LMAN2 in brain metastatic breast cancer. We again found that LMAN2 was among the genes whose expression changed most significantly when comparing metastases of the brain to primary tumors of the breast in patients with breast cancer (Table 2). When sorting each of the genes expressed in brain metastases based on significance of change as compared to primary tumors of the breast, LMAN2 ranked 1380 out of 61359 total transcripts (Table 2). Differential expression of LMAN2 in brain metastases in brain metastatic breast cancer was statistically significant (Table $2 ; \mathrm{p}=0.0081642$ ). 
LMAN2 is expressed at significantly higher levels in brain metastases as compared to primary tumors of the breast in brain metastatic breast cancer.

We obtained exact mRNA expression values for LMAN2 from brain metastases and primary tumors of the breast to evaluate magnitude and direction of change in LMAN2 expression in brain metastases in patients with breast cancer. LMAN2 was expressed at higher levels in brain metastases in patients with breast cancer and this difference was statistically significant (Figure $1 ; p=0.0046$ ). We calculated a mean fold change of $1.138 \pm 0.028$ in LMAN2 expression when comparing brain metastatic tissues to primary tumors of the breast (Table 1).

Thus, we found that LMAN2 was among the genes, globally, whose expression was most different when comparing primary tumors of the breast to brain metastases in patients with breast cancer.

\section{Discussion}

An enhanced understanding of brain metastases can facilitate development of novel therapeutic strategies. We identified the L-type lectin LMAN24-7 as among the genes most differentially expressed in brain metastases when compared to primary tumors of the breast in patients with breast cancer. As we found LMAN2 was over-expressed in primary tumors of the breast, and since LMAN2 has a structurallydefined carbohydrate-recognition domain 7 , future studies can evaluate whether chemical inhibition or blockade of the carbohydrate-recognition domain of LMAN2 is therapeutically beneficial in animal models of metastatic breast cancer or in human patients with brain metastatic breast cancer. 


\section{References}

1. Bendell, J.C., Domchek, S.M., Burstein, H.J., Harris, L., Younger, J., Kuter, I., Bunnell, C., Rue, M., Gelman, R. and Winer, E., 2003. Central nervous system metastases in women who receive trastuzumab-based therapy for metastatic breast carcinoma. Cancer, 97(12), pp.2972-2977.

2. Schulten, H.J., Bangash, M., Karim, S., Dallol, A., Hussein, D., Merdad, A., Al-Thoubaity, F.K., AlMaghrabi, J., Jamal, A., Al-Ghamdi, F. and Choudhry, H., 2017. Comprehensive molecular biomarker identification in breast cancer brain metastases. Journal of translational medicine, 15(1), p.269.

3. McMullin, R.P., Wittner, B.S., Yang, C., Denton-Schneider, B.R., Hicks, D., Singavarapu, R., Moulis, S., Lee, J., Akbari, M.R., Narod, S.A. and Aldape, K.D., 2014. A BRCA1 deficient-like signature is enriched in breast cancer brain metastases and predicts DNA damage-induced poly (ADP-ribose) polymerase inhibitor sensitivity. Breast Cancer Research, 16(2), pp.1-10.

4. Kwon, S.H., Oh, S., Nacke, M., Mostov, K.E. and Lipschutz, J.H., 2016. Adaptor protein CD2AP and L-type lectin LMAN2 regulate exosome cargo protein trafficking through the Golgi complex. Journal of Biological Chemistry, 291(49), pp.25462-25475.

5. Fiedler, K., Parton, R.G., Kellner, R., Etzold, T. and Simons, K., 1994. VIP36, a novel component of glycolipid rafts and exocytic carrier vesicles in epithelial cells. The EMBO journal, 13(7), pp. 1729-1740.

6. Kamiya, Y., Yamaguchi, Y., Takahashi, N., Arata, Y., Kasai, K.I., Ihara, Y., Matsuo, I., Ito, Y., Yamamoto, K. and Kato, K., 2005. Sugar-binding properties of VIP36, an intracellular animal lectin operating as a cargo receptor. Journal of Biological Chemistry, 280(44), pp.37178-37182.

7. Satoh, T., Cowieson, N.P., Hakamata, W., Ideo, H., Fukushima, K., Kurihara, M., Kato, R., Yamashita, K. and Wakatsuki, S., 2007. Structural basis for recognition of high mannose type glycoproteins by mammalian transport lectin VIP36. Journal of Biological Chemistry, 282(38), pp.28246-28255.

8. Robson, M., Im, S.A., Senkus, E., Xu, B., Domchek, S.M., Masuda, N., Delaloge, S., Li, W., Tung, N., Armstrong, A. and Wu, W., 2017. Olaparib for metastatic breast cancer in patients with a germline BRCA mutation. New England Journal of Medicine, 377(6), pp.523-533.

PAGE 4 


\begin{tabular}{|c|c|c|c|c|c|c|c|}
\hline Rank & ID & p-value & $\mathbf{t}$ & B & FC & Gene & Gene name \\
\hline 87 & 8116020 & 4.99E-07 & 7.2551264 & 6.462268 & $1.138 \pm 0.028$ & LMAN2 & $\begin{array}{l}\text { lectin, mannose } \\
\text { binding } 2\end{array}$ \\
\hline
\end{tabular}

\section{Table 1: LMAN2 is differentially expressed in brain metastatic breast cancer.}

Rank of differential expression, probe ID, $p$-value with respect to differential expression, $t$, a moderated tstatistic, B, the log-odds of differential expression between the two groups compared, fold change (FC) of LMAN2 expression between the brain metastases of patients with breast cancer and primary tumors of the breast, gene and gene name are listed in this chart. 


\begin{tabular}{r|l|l|l|l|l|l}
\hline Rank & ID & p-value & t & B & Gene & Gene name \\
\hline 1380 & 215656_3p_at & 0.0081642 & -2.7742036 & -2.4999 & LMAN2 & $\begin{array}{l}\text { lectin, mannose } \\
\text { binding 2 }\end{array}$ \\
\hline
\end{tabular}

\section{Table 2: LMAN2 is differentially expressed in brain metastatic breast cancer.}

Rank of differential expression, probe ID, $p$-value with respect to differential expression, $\mathrm{t}$, a moderated $\mathrm{t}$ statistic, B, the log-odds of differential expression between the two groups compared, gene and gene name are listed in this chart. 


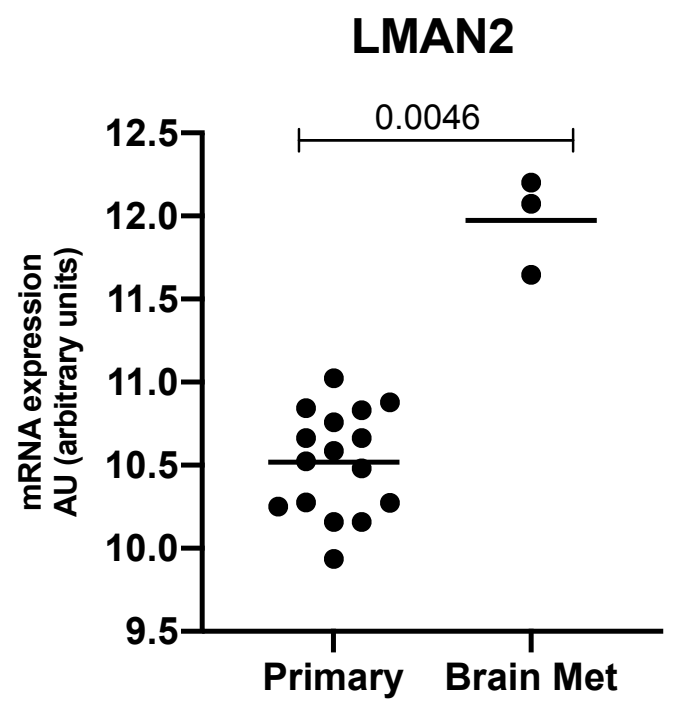

Figure 1: LMAN2 is expressed at significantly higher levels in brain metastases as compared to primary tumors of the breast in patients with brain metastatic breast cancer.

The mRNA expression level of LMAN2 in primary tumors of the breast (left) and in the brain metastases of brain metastatic breast cancer patients (right) is graphically represented here with mean LMAN2 levels marked and the result of a statistical test evaluating difference in mRNA expression level between brain metastases and primary tumors of the breast, a $p$-value, listed above. 